\title{
Non-perturbative renormalization of bilinear operators with Möbius domain-wall fermions in the coordinate space
}

\author{
JLQCD Collaboration: M. Tomii ${ }^{* a, b}$, G. Cossu ${ }^{b}$, S. Hashimoto ${ }^{a, b}$, J. Noaki ${ }^{b}$ \\ E-mail: tomiidpost.kek.ip \\ ${ }^{a}$ School of High Energy Accelerator Science, The Graduate University for Advanced Studies \\ (Sokendai), Ibaraki 305-0801, Japan, \\ ${ }^{b}$ KEK Theory Center, Institute of Particle and Nuclear Studies, High Energy Accelerator \\ Research Organization (KEK), Ibaraki 305-0801, Japan
}

\begin{abstract}
We study the non-perturbative determination of the renormalization constants of flavor non-singlet quark bilinear operators on the lattice. The renormalization condition is imposed on correlation functions of bilinear operators in the coordinate space. The results are converted to the value at $2 \mathrm{GeV}$ in the $\overline{\mathrm{MS}}$ scheme by a perturbative matching. The calculation is carried out on gauge configurations generated with the Mobius domain-wall fermions at two lattice spacings $a^{-1}=2.4$ $\mathrm{GeV}$ and $a^{-1}=3.6 \mathrm{GeV}$.
\end{abstract}

The 32nd International Symposium on Lattice Field Theory,

23-28 June, 2014

Columbia University New York, NY

${ }^{*}$ Speaker. 


\section{Introduction}

There are several methods to renormalize the lattice operators onto those defined in the continuum renormalization scheme such as the $\overline{\mathrm{MS}}$ scheme. Schrödinger functional [W] and RI/MOM [ $\square]$ approaches are among the popular choices. The former provides precise and fully non-perturbative renormalization at the cost of generating dedicated ensembles. The renormalization condition in the latter approach is imposed on the vertex function at a fixed gauge and it involves (continuum) perturbative expansion which is calculated only at one- or two-loop order.

In this work, we investigate the determination of the renormalization constants by the $\mathrm{X}$-space method, which was originally suggested in [B] and has been developed in [ [ the renormalization condition is imposed on two-point correlation functions of the operators to be renormalized. Unlike RI/MOM, the X-space method enables us to renormalize by a gauge invariant quantity. By keeping the distance finite, one can avoid extra divergences due to contact terms. Another important advantage is that the perturbative expansion on the continuum side is available to the four-loop level for the quark bilinear operators [焑]. On the other hand, a potential problem of the X-space method is the requirement for a "window" where the continuum perturbation theory can be applied and the lattice calculation with minimal discretization effect is possible.

In this report, we present the preliminary results of the determination of renormalization constants of quark non-singlet bilinear operators using the $\mathrm{X}$-space method. We obtain good precision on the lattices generated with the $2+1$ flavors of Möbius domain-wall fermions and Symanzik improved gauge action $[\square]$. We work on $32^{3} \times 64$ lattices at $a^{-1}=2.4 \mathrm{GeV}$ and $48^{3} \times 96$ lattices at $a^{-1}=3.6 \mathrm{GeV}$, both having matched physical volume and similar input physical masses in the window of $M_{\pi}: 300 \sim 500 \mathrm{MeV}$.

\section{Sketch of the $\mathrm{X}$-space method}

We impose the renormalization condition on massless correlation functions of quark (nonsinglet) bilinear operators, which are defined as

$$
\begin{aligned}
& \Pi_{\mathrm{SS}}(x)=\langle S(x) S(0)\rangle, \quad \Pi_{\mathrm{PP}}(x)=\langle P(x) P(0)\rangle \\
& \Pi_{\mathrm{VV}}(x)=\sum_{\mu=1}^{4}\left\langle V_{\mu}(x) V_{\mu}(0)\right\rangle, \quad \Pi_{\mathrm{AA}}(x)=\sum_{\mu=1}^{4}\left\langle A_{\mu}(x) A_{\mu}(0)\right\rangle,
\end{aligned}
$$

where $S$ and $P$ are scalar and pseudoscalar densities, $V_{\mu}$ and $A_{\mu}$ are vector and axial-vector currents. Flavor indices are omitted for simplicity, but they are understood as isospin triplet operators of light quarks. These bilinear operators on the lattice are to be renormalized onto the $\overline{\mathrm{MS}}$ scheme at $2 \mathrm{GeV}$, i.e.

$$
\left.O_{\Gamma}^{\overline{\mathrm{MS}}}\right|_{2 \mathrm{GeV}}=\left.Z_{\Gamma}^{\overline{\mathrm{MS}} / l a t}(2 \mathrm{GeV} ; a) O_{\Gamma}^{\text {lat }}\right|_{a},
$$

for each quark bilinear operators $O_{\Gamma} \in\left\{S, P, V_{\mu}, A_{\mu}\right\}$.

Since these correlation functions have two bilinear operators, the renormalization condition is given by

$$
\left.Z_{\Gamma}^{\overline{\mathrm{MS}} / \mathrm{lat}}(2 \mathrm{GeV} ; a)^{2} \Pi_{\Gamma \Gamma}^{\mathrm{lat}}(x)\right|_{a}=\left.\Pi_{\Gamma \Gamma}^{\overline{\mathrm{MS}}}(x)\right|_{2 \mathrm{GeV}},
$$




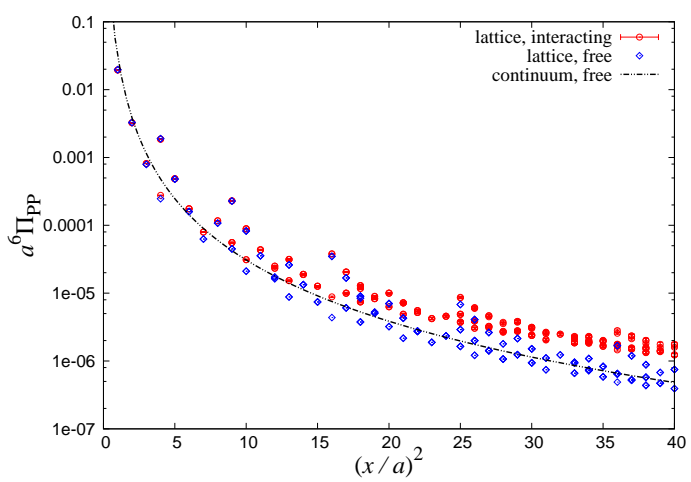

Figure 1: Correlation function for $\mathrm{P}$ channel which are purely measured on the lattice in the ensemble $48^{3} \times 96, \beta=4.35, a m_{u d}=0.012, a m_{s}=$ 0.018 and that in the free system plotted with that in free continuum theory.

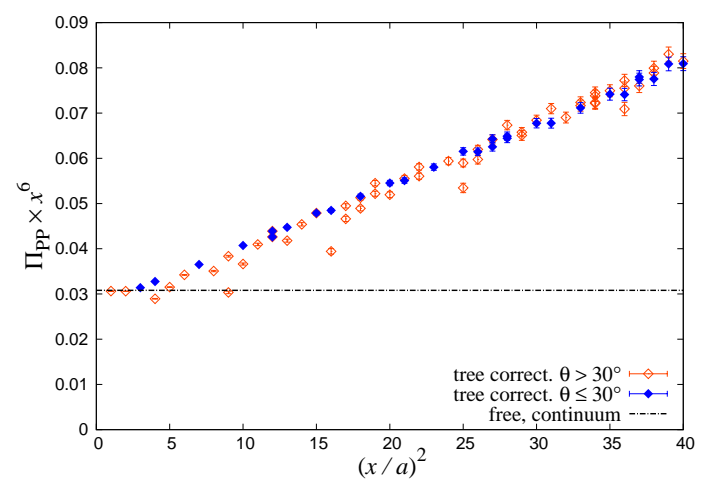

Figure 2: Correlation function after applying treelevel correction to that in Fig. 1 with the discrimination of the range $\theta>30^{\circ}$ (open diamond) from $\theta \leq 30^{\circ}$ (filled diamond).

or

$$
Z_{\Gamma}^{\overline{\mathrm{MS}} / l a t}(2 \mathrm{GeV} ; a)=\sqrt{\frac{\left.\Pi_{\Gamma \Gamma}^{\overline{\mathrm{MS}}}(x)\right|_{2 \mathrm{GeV}}}{\left.\Pi_{\Gamma \Gamma}^{\text {lat }}(x)\right|_{a}}},
$$

where $\Pi_{\Gamma \Gamma}^{\overline{\mathrm{MS}}}$ and $\Pi_{\Gamma \Gamma}^{\text {lat }}$ are correlation functions in the continuum theory or measured on the lattice.

We perform two calculations to obtain the renormalization constants:

- $\left.\Pi_{\Gamma \Gamma}^{\text {lat }}(x)\right|_{a}$ : Correlation functions calculated on the lattice and taken the chiral limit.

- $\left.\Pi_{\Gamma \Gamma}^{\overline{\mathrm{MS}}}(x)\right|_{2 \mathrm{GeV}}$ : Massless continuum correlation functions renormalized at $2 \mathrm{GeV}$ in the $\overline{\mathrm{MS}}$ scheme.

In this process, we should choose $x$ in the window $a \ll|x| \ll \Lambda_{\mathrm{QCD}}^{-1}$ in order to avoid discretization effect and perturbative ambiguity.

\section{Correlation functions measured on the lattice}

Figure $\amalg$ shows $x^{2}$-dependence of short-distance lattice correlator of pseudoscalar channel plotted as a function of $x^{2}$ (red). In this plot, we also show the two-point correlator calculated at the tree-level, i.e. no strong interaction (blue). The data show substantial violation of the rotational symmetry, but apparently their short-distance behavior is rather precisely reproduced by the free propagator. The simplest example is the two-points at $(x / a)^{2}=4$ where $(2,0,0,0)$ and $(1,1,1,1)$ are different 10-times, but they are well exploited at tree-level.

We can thus eliminate the bulk of discretization effect by subtracting the tree-level contribution of the discretization effect as

$$
\Pi_{\Gamma \Gamma}^{\text {lat }}(x) \longrightarrow \Pi_{\Gamma \Gamma}^{\text {lat }}(x)-\left(\Pi_{\Gamma \Gamma}^{\text {lat, free }}(x)-\Pi_{\Gamma \Gamma}^{\text {cont }, \text { free }}(x)\right) .
$$

The results are shown in Fig. $\square$. We note that the vertical axis in the linear scale rather than logarithmic. Reductions of the Lorentz violation is quite clear. A similar subtraction was introduced, but 

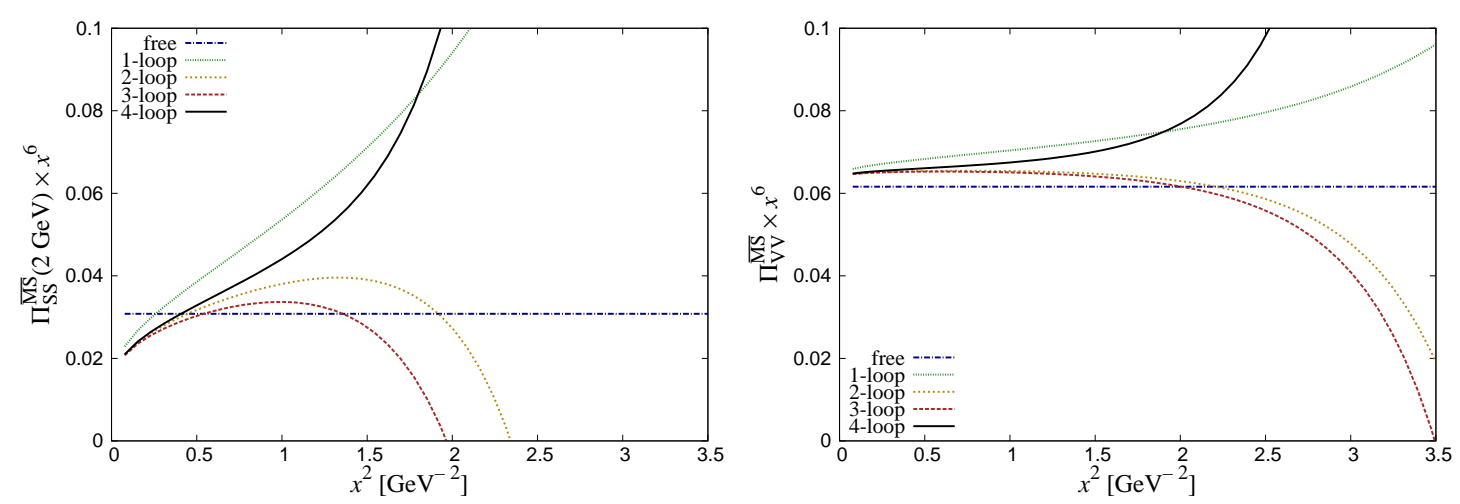

Figure 3: Massless correlators from perturbation theory with $N_{f}=3, \Lambda_{\mathrm{QCD}}=340 \mathrm{MeV}$, scalar channel at $2 \mathrm{GeV}$ in $\overline{\mathrm{MS}}$ scheme (left panel) and vector channel (right panel).

multiplicatively, i.e. multiplying the original correlator $\Pi_{\Gamma \Gamma}^{\text {lat }}(x)$ by the ratio $\Pi_{\Gamma \Gamma}^{\text {cont } \text { free }}(x) / \Pi_{\Gamma \Gamma}^{\text {lat, free }}(x)$. We find that (B. W) works slightly better to eliminate the Lorentz violating effect.

Another way to reduce discretization effect is suggested [[] on top of (B.D). Let us define $\theta$ as an angle between $x$ and the direction $(1,1,1,1)$. One may find that the discretization effect becomes more pronounced for large $\theta$. By inspecting the results, we decided to drop the points of $\theta>30^{\circ}$. In Fig. \, the discarded points are shown by open symbols. The remaining points (blue diamonds) are quite smooth as a function of $(x / a)^{2}$.

\section{Correlation functions computed from continuum perturbation theory}

Perturbative expansion of the massless correlators in the $\overline{\mathrm{MS}}$ scheme

$$
\begin{aligned}
& \Pi_{\mathrm{SS}, \mathrm{PP}}^{\overline{\mathrm{MS}}}(x, \mu)=\Pi_{\mathrm{SS}, \mathrm{PP}}^{\widetilde{\mathrm{MS}}}(x, \tilde{\mu})=\frac{3}{\pi^{4} x^{6}}\left(1+\sum_{n} \widetilde{C}_{n}^{\mathrm{S}} \tilde{a}_{s}^{n}\right), \\
& \Pi_{\mathrm{VV}, \mathrm{AA}}^{\overline{\mathrm{MS}}}(x)=\Pi_{\mathrm{VV}, \mathrm{AA}}^{\widetilde{\mathrm{MS}}}(x)=\frac{6}{\pi^{4} x^{6}}\left(1+\sum_{n} \widetilde{C}_{n}^{\mathrm{V}} \tilde{a}_{s}^{n}\right), \\
& \tilde{a}_{s}=\frac{\alpha_{s}^{\widetilde{\mathrm{MS}}}(\tilde{\mu}=1 / x)}{\pi}=\frac{\alpha_{s}^{\overline{\mathrm{MS}}}\left(\mu=2 \mathrm{e}^{-\gamma_{E}} / x\right)}{\pi},
\end{aligned}
$$

are known up to $n=4$ [目]. $\widetilde{C}_{n}^{\mathrm{S}, \mathrm{V}}$ are perturbative coefficients and $a_{s}=\alpha_{s} / \pi$. The running of $\alpha_{s}$ is also known up to four-loop level [8] in the $\overline{\mathrm{MS}}$ scheme.

Since the scale $\mu$ and $\tilde{\mu}$ in these formulae depend on $x$, we need to perform the scale evolution for correlation functions of scalar and pseudoscalar correlators to those at $2 \mathrm{GeV}$ in the $\overline{\mathrm{MS}}$ scheme. The scale evolution is calculated as

$$
\Pi_{\mathrm{SS}, \mathrm{PP}}^{\widetilde{\mathrm{MS}}}\left(x, \tilde{\mu}_{1}\right)=\left[\frac{c\left(a_{s}\left(\tilde{\mu}_{1}\right)\right)}{c\left(a_{s}\left(\tilde{\mu}_{0}\right)\right)}\right]^{-2} \Pi_{\mathrm{SS}, \mathrm{PP}}^{\widetilde{\mathrm{MS}}}\left(x, \tilde{\mu}_{0}\right), \quad c(x) \equiv \exp \left[\int^{x} \mathrm{~d} x^{\prime} \frac{\gamma_{m}\left(x^{\prime}\right)}{\beta\left(x^{\prime}\right)}\right],
$$

where $\gamma_{m}$ and $\beta$ are the quark mass anomalous dimension and the QCD beta function, and $c(x)$ is given in [Q, [0] $]$. The Ward-Takahashi identity guarantees that the correlation functions of vector or axial-vector currents are scale independent. 

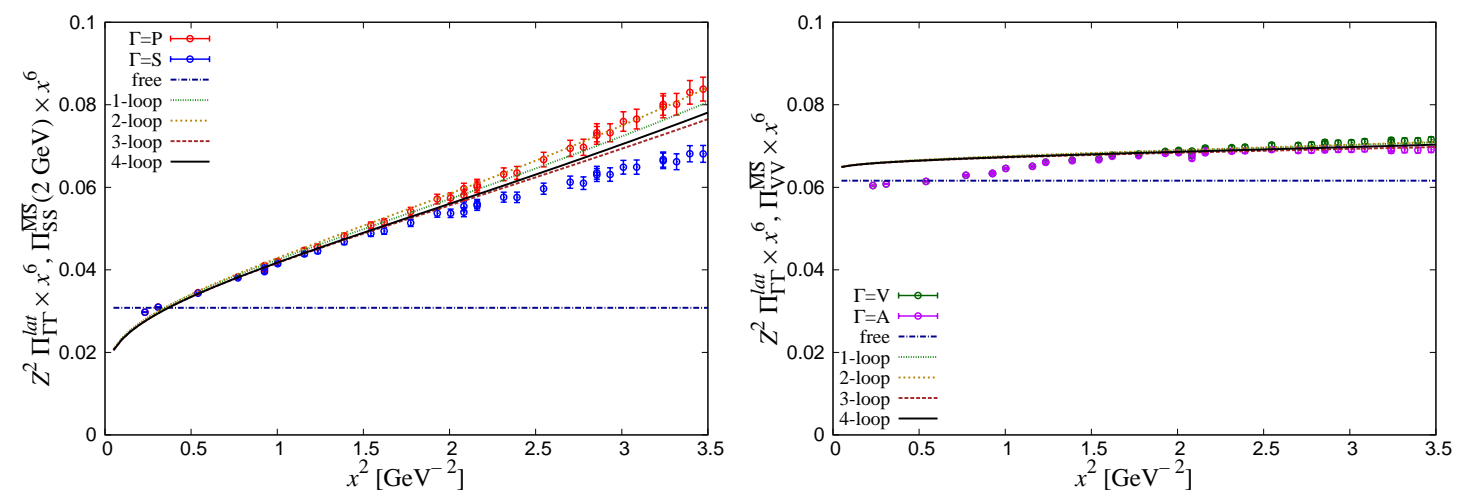

Figure 4: Massless correlators from perturbation theory with improved convergence and chiral limits of lattice correlators times some constants, which are squares of renormalization constants. These lattice correlators are measured on $48^{3} \times 96, a^{-1}=3.6 \mathrm{GeV}$ lattice, while a sea quark is still massive $a m_{s}=0.0180$.

As shown in Fig. B, the convergence of the perturbative series in (4. (ل) and (4.2) is not sufficiently good to achieve precise determination of the renormalization constant already at $x \simeq$ $1 \mathrm{GeV}^{-1}$. At longer distances, the expansion is even divergent. the perturbative series in $N_{f}=3$ theory are given as

$$
\begin{aligned}
& \Pi_{\mathrm{SS}}^{\widetilde{\mathrm{MS}}}(x, \tilde{\mu})=\frac{3}{\pi^{4} x^{6}}\left(1+0.67 \tilde{a}_{s}-16.3 \tilde{a}_{s}^{2}-31 \tilde{a}_{s}^{3}+497 \tilde{a}_{s}^{4}\right), \\
& \Pi_{\mathrm{VV}}^{\widetilde{\mathrm{MS}}}(x)=\frac{6}{\pi^{4} x^{6}}\left(1+\tilde{a}_{s}-4 \tilde{a}_{s}^{2}-1.9 \tilde{a}_{s}^{3}+94 \tilde{a}_{s}^{4}\right) .
\end{aligned}
$$

When $\tilde{a}_{s}=0.1$, the expansion for SS is like $1+0.067-0.163-0.031+0.050$ and does not seem to converge.

In order to avoid this problem, we expand correlators in terms of the coupling $a_{s}^{*}$ at another scale $\mu^{*}$ according to the BLM prescription [W]. In this way, one can effectively absorbs higherorder contribution from vacuum polarization effects into lower-orders. The scale for VV (and AA) is given by $\mu^{*}=\mu \mathrm{e}^{-11 / 6+2 \zeta(3)} \simeq 1.8 \mu$. We use the same scale also for SS (and PP).

The resulting expansion becomes

$$
\begin{aligned}
& \Pi_{\mathrm{SS}}^{\widetilde{\mathrm{MS}}}\left(x, \mu^{*}\right)=\frac{3}{\pi^{4} x^{6}}\left(1+2.9 a_{s}^{*}+1.1 a_{s}^{* 2}-42 a_{s}^{* 3}+24 a_{s}^{* 4}\right), \\
& \Pi_{\mathrm{VV}}^{\widetilde{\mathrm{MS}}}(x)=\frac{6}{\pi^{4} x^{6}}\left(1+a_{s}^{*}+0.083 a_{s}^{* 2}-6 a_{s}^{* 3}+18 a_{s}^{* 4}\right),
\end{aligned}
$$

which obviously has much better convergence property. The scale evolution for $\Pi_{S S}$ to $2 \mathrm{GeV}$ in $\overline{\mathrm{MS}}$ is then calculated using (4.4).

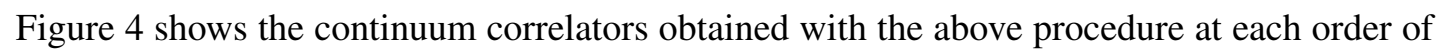
the perturbative expansion. It clearly shows a good convergence even at lower scales $x^{2} \sim 3 \mathrm{GeV}^{-2}$.

\section{Preliminary results}

Figure $⿴$ also shows the lattice data rescaling by a factor such that they agree with the perturbation theory in the region of $x^{2} \sim 2 \mathrm{GeV}^{-2}$. This factor corresponds to the renormalization factor 


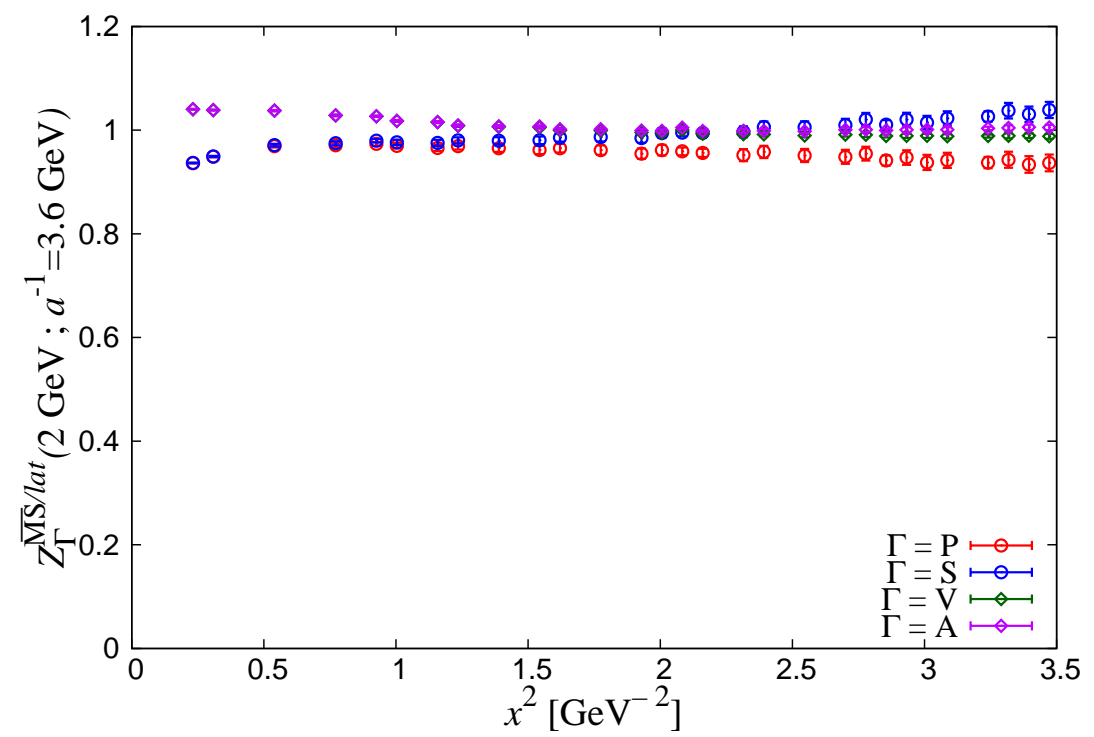

Figure 5: Renormalization constants with the dependence on the renormalization point $x$, where the parameters are same as Fig. 4.

squared as given in (2.4).

Figure $\square$ shows the renormalization constant calculated from eq. ([2.5]) with dependence on the renormalization point $x$. Since LHS of eq. (2.5) is independent of $x$, we should extract RCs from a range where $x$-dependence of RCs is approximately absent. Since we employ the Möbius of domain wall fermions which have excellent chiral symmetry, we can assume $Z_{\mathrm{S}}=Z_{\mathrm{P}}, Z_{\mathrm{V}}=Z_{\mathrm{A}}$ in the chiral limit, and extract $Z_{\mathrm{S}}$ and $Z_{\mathrm{V}}$ as an average of $\left\{Z_{\mathrm{S}}, Z_{\mathrm{P}}\right\}$ or $\left\{Z_{\mathrm{V}}, Z_{\mathrm{A}}\right\}$, respectively. Here the difference between $Z_{\mathrm{S}}$ and $Z_{\mathrm{P}}$, or $Z_{\mathrm{V}}$ and $Z_{\mathrm{A}}$, coming from higher orders of the operator product expansion is considered as a part of systematic errors.

Table U shows the preliminary results for RCs. Since we calculate only at two strange quark masses for each lattice spacings, we don't take the chiral limit of $m_{s}$. Systematic errors, which are the second errors in Tab. $\mathrm{W}$, are typically $1 \%$ or less except for $Z_{\mathrm{S}}$ at $a^{-1}=2.4 \mathrm{GeV}$ lattice with $a m_{s}=0.040$. This $1 \%$ precision for $Z_{\mathrm{S}}$ is better than that of other methods, such as RI/MOM at similar lattice spacings.

Table 1: Preliminary results of computing renormalization constants. First error means statistical error and second one means systematic error.

\begin{tabular}{cccc}
\hline \hline$a^{-1}[\mathrm{GeV}]$ & $a m_{s}$ & $Z_{S}^{\overline{\mathrm{MS}} / \text { lat }}(2 \mathrm{GeV})$ & $Z_{V}^{\overline{\mathrm{MS}} / \text { lat }}$ \\
\hline 2.4 & 0.030 & $1.092(6)(12)$ & $1.013(2)(7)$ \\
2.4 & 0.040 & $1.093(8)(28)$ & $1.017(2)(9)$ \\
\hline 3.6 & 0.018 & $0.973(5)(6)$ & $0.999(3)(5)$ \\
3.6 & 0.025 & $0.965(7)(11)$ & $0.994(6)(5)$ \\
\hline \hline
\end{tabular}




\section{Summary}

We investigate the Non-perturbative renormalization of flavor non-singlet quark bilinear operators using the gauge invariant X-space method for the action of $2+1$ Möbios domain-wall fermions and Symanzik improved gauge action.

Discretization effect of correlation functions measured on lattice are mostly reduced by applying the tree-level correction and the democratic cut. On the other hand, convergence of perturbation theory is sufficiently improved by expanding correlators into a polynomials of coupling $a_{s}^{*}$ at an appropriate scale $\mu^{*}$.

The systematic error we reach is roughly within $1 \%$, which has been difficult systematic precision for renormalization constants of scalar and pseudoscalar dencities. Therefore the X-space method may be a very useful way of the non-perturbative renormalization.

\section{Acknowledgments}

Numerical simulations are performed on the IBM System Blue Gene Solution at High Energy Accelerator Research Organization (KEK) under a support of its Large Scale Simulation Program (No. 13/14-04). This work is supported in part by the Grant-in-Aid of the Japanese Ministry of Education (Nos. ${ }^{* *}$ ) and the SPIRE (Strategic Program for Innovative Research) Field5 project.

\section{References}

[1] M. Lüscher, R. Narayanan, P. Weisz, and U. Wolff, Nucl. Phys. B 384 (1992) 168, hep-lat/9207009.

[2] G. Martinelli, C. Pittori, C.T. Sachrajda, M. Testa, and A. Vladikas, Nucl. Phys. B 445 (1995) 81, hep-lat/9411010.

[3] G. Martinelli, G.C. Rossi, C.T. Sachrajda, S.R. Sharpe, M. Talevi, M. Testa, Phys. Lett. B 411 (1997) 141, hep-lat/9705018.

[4] V. Giménez, L. Giusti, S. Guerriero, V. Lubicz, G. Martinelli, S. Petrarca, J. Reyes, B. Taglienti, and E. Trevigne, Phys. Lett. B 598 (2004) 227, hep-lat/ 0406019.

[5] K. Cichy, Karl Jansen, and Piotr Korcyl, Nucl. Phys. B 865 (2012) 268, arXiv: 1207.0628 [hep-lat].

[6] K.G. Chetyrkin and A. Maier, Nucl. Phys. B 844 (2011) 266, arXiv: 1010.1145 [hep-ph] .

[7] J. Noaki, S. Aoki, G. Cossu, H. Fukaya, S. Hashimoto and T. Kaneko, (Now applying to PoS LATTICE 2014 (2014) ***).

[8] K.G. Chetyrkin, B.A. Kniehl, and M. Steinhauser, Phys. Rev. Lett. 79 (1997) 2184, hep-ph/9706430.

[9] K. G. Chetyrkin, Phys. Lett. B 404 (1997) 161, hep-ph / 9703278.

[10] J.A.M. Vermaseren, S.A. Larin, and T. van Ritbergen, Phys. Lett. B 405 (1997) 327, hep-ph/9703284.

[11] S. J. Brodsky, G. P. Lepage and P. B. Mackenzie, Phys. Rev. D 28 (1983) 228. 